\title{
Direct Observation of Cationic Ordering in Double Perovskite $\mathrm{Sr}_{2} \mathrm{FeReO}_{6}$ Crystals
}

\author{
Si-Young Choi, ${ }^{1, \star}$ Jong Bong Lim, ${ }^{2}$ Yuichi Ikuhara, ${ }^{3}$ Danilo Suvorov, ${ }^{4}$ and Jae-Ho Jeon ${ }^{2}$ \\ ${ }^{1}$ Advanced Characterization and Analysis Group, Korea Institute of Materials Science, Changwon 641-831, \\ Republic of Korea \\ ${ }^{2}$ Nano-Functional Materials Group, Korea Institute of Materials Science, Changwon 641-831, Republic of Korea \\ ${ }^{3}$ Institute of Engineering Innovation, The University of Tokyo, Tokyo 113-8656, Japan \\ ${ }^{4}$ Department of Advanced Materials, Jozef Stefan Institute, Ljubljana SI-1000, Slovenia
}

\begin{abstract}
Two kinds of $\mathrm{Sr}_{2} \mathrm{FeReO}_{6}$ (SFRO) samples, pristine SFRO and Re-excess SFRO, were prepared and we visualized the local atomic structure in terms of cationic ordering in the prepared SFRO samples via high-angle annular dark-field (HAADF) scanning transmission electron microscopy (STEM). HAADF-STEM results demonstrated the high degree of cationic ordering maintains in both the pristine SFRO and Re-excess SFRO samples. On the other hand, defective structures such as antiphase boundary and Re-deficient phase were observed dominantly in the pristine SFRO, and thus the poor magnetic property in the pristine SFRO is attributed to those defective structures related with the frustrated Fe/Re ordering.
\end{abstract}

Key words: double perovskite, cationic ordering, scanning transmission electron microscopy, high-angle annular dark-field

\section{INTRODUCTION}

Cation ordering double perovskites, having the typical formula $\mathrm{A}_{2} \mathrm{BB}^{\prime} \mathrm{O}_{6}$ where $\mathrm{A}$ is an alkaline earth cation and $\mathrm{B}$ and $\mathrm{B}^{\prime}$ are heterovalent transition metals, are half-metallic magnetic oxides that have been attracting a great deal of attention in the field of spin-polarized transport applications. Moreover, these double perovskites have become one of a group of promising magnetic materials for spin electronics because of their high $T_{\mathrm{c}}$, the value of which is much higher than $300 \mathrm{~K}$, and their tunneling-type magnetoresistance (Kobayashi et al., 1998; Fang et al., 2001). The magnetic structure of $\mathrm{A}_{2} \mathrm{BB}^{\prime} \mathrm{O}_{6}$ is known to originate from the ordered arrangement of parallel $\mathrm{Fe}^{3+}\left(3 \mathrm{~d}^{5}, \mathrm{~S}=5 / 2\right)$ magnetic moments, antiferromagnetically coupled with $\mathrm{Mo}^{5+}\left(4 \mathrm{~d}^{1}\right.$, $\mathrm{S}=1 / 2)$ spins or $\operatorname{Re}^{5+}\left(5 \mathrm{~d}^{2}, \mathrm{~S}=1\right)$ spins. These oxides present antisite (AS) defects, in which cationic ordering in $\mathrm{B}$-sites is frustrated by the occupation of $\mathrm{B}$ cations of $\mathrm{B}^{\prime}$ sites and vice versa; this situation is known to remarkably degrade the magnetic saturation, as well as the magnetoresistance (Balcells et al., 2001; García-Hernádez et al., 2001; Navarro et al., 2001).

So far, the conventional way to determine the presence of cationic ordering such as AS defects is to utilize a statistical tool (Lindén et al., 2000; Nakamura \& Oikawa, 2003; Zajac et al., 2007) such as neutron diffraction, X-ray diffraction (XRD), and Mossbauer spectroscopy, but the atomicscale observation of the local distribution of $\mathrm{Fe} / \mathrm{Re}$ disordering cannot be predicted by such macroscopic analytical tools, even though the atomic-scale defective structures, such as antiphase boundary (APB) and antiferromagnetic $\mathrm{Fe}-\mathrm{O}-\mathrm{Fe}$ islands, have been suggested to diminish the mag-

(c) MICROSCOPY SOCIETY OF AMERICA 2013

${ }^{\star}$ Corresponding author. E-mail: youngchoi@kims.re.kr netic property (Lindén et al., 2000; Yin et al., 2000; GarcíaHernádez et al., 2001; Navarro et al., 2001). In this study, we utilized scanning transmission electron microscopy (STEM) with a spherical aberration corrector to visualize the cationic ordering in $\mathrm{Sr}_{2} \mathrm{FeReO}_{6}$ (SFRO), as the correction of aberrations in electron microscopy (Haider et al., 1998; Batson et al., 2002; Nellist et al., 2004; Jia et al., 2008) has allowed the resolution of images to be significantly enhanced to the subangstrom level and furthermore facilitates the observation of sensitive variations in atomic column images (Choi et al., 2009; Chung et al., 2009).

\section{Methods and Materials}

The pristine SFRO powder was first synthesized by the conventional solid-state method, using $\mathrm{SrCO}_{3}, \mathrm{Fe}_{2} \mathrm{O}_{3}$, and $\mathrm{ReO}_{3} ; 15$ wt\%-Re-excess SFRO powder was also prepared using the same starting materials, along with $\mathrm{ReO}_{3}$. The mixed powders were pressed into discs and calcined at $900^{\circ} \mathrm{C}$ for $3 \mathrm{~h}$ under Ar atmosphere. All calcined powders were charged into $10-\mathrm{mm}$-diameter graphite molds and then sintered at $1,100^{\circ} \mathrm{C}$ for 10 min by using spark plasma sintering (SPS-1050; Sumitomo Coal Mining) under 65 $\mathrm{MPa}$ of pressure in $\mathrm{Ar}$ atmosphere. The sintered bodies were followed by annealing in air at $700^{\circ} \mathrm{C}$ for $0.5 \mathrm{~h}$ to remove the contamination of carbon on the sample surfaces.

High-angle annular dark-field (HAADF) images were recorded with an STEM (JEM-2100F, JEOL) at $200 \mathrm{kV}$ with a spherical aberration corrector (CEOS GmbH), and thus the size of the electron probe was $\sim 1.0 \AA$. In the HAADF imaging, a HAADF detector with an inner angle greater than $\sim 80 \mathrm{mrad}$ was used in order to allow for large-angle elastic scattering of electrons for clear Z-sensitive images. The obtained raw images were filtered to eliminate back- 
ground noise using 2D difference filters (HREM Research Inc.). Image simulations based on the multislice method were performed using WinHREM (HREM Research Inc.).

\section{Results AND Discussion}

Magnetization-magnetic field hysteresis loops indicated that the value of the saturated magnetization $\left(M_{\mathrm{s}}\right)$ of pristine SFRO was found to deteriorate down to $14.3 \mathrm{emu} / \mathrm{g}$, compared with Re-excess SFRO (21.4 emu/g). Moreover, the pristine SFRO sample can be saturated at a higher magnetic field and, accordingly, can exhibit a larger coercive magnetic field $\left(H_{\mathrm{c}}\right)$. Lower $M_{\mathrm{s}}$ and higher $H_{\mathrm{c}}$ in the pristine SFRO sample reveals that the ferromagnetic alignment of Fe and $\mathrm{Re}$ atoms is interfered with by the $\mathrm{Fe} / \mathrm{Re}$ disordering. Before an atomic-scale analysis, the pristine and Re-excess SFRO samples were analyzed with an XRD diffractometer. It was found that $\mathrm{Fe} / \mathrm{Re}$ disordering structure in the pristine SFRO was determined to reach up to $\sim 15 \%$, whereas the Reexcess SFRO showed a much higher degree of Fe/Re ordering, with a value of $<3 \%$. Therefore, the magnetic property and the XRD results demonstrated that the magnetic saturation was diminished by the $\mathrm{Fe} / \mathrm{Re}$ disordering.

For both the pristine and the Re-excess SFRO samples, the HAADF-STEM images from the [001] and [110] projections are shown in Figures 1a and 1b, respectively. Although only two atomic columns of the $\mathrm{Fe}$ and Re column (orange sphere) and the Sr column (green sphere) were distinguished in the [001] projection, three kinds of atomic columns of the Fe column (yellow sphere), the Re column (blue sphere), and the Sr column (green sphere) can be clearly noticed in the [110] projection, indicating that the $\mathrm{Fe} / \mathrm{Re}$ ordering in SFRO can be confirmed on an atomic scale in the [110] projection. Figure 1c includes three intensity profiles of columns along from the pristine SFRO (red line), the Re-excess SFRO (blue line), and the simulated image (green symbols). Although the HAADF-STEM image simulation was carried out based on the defect-free SFRO structure and the pristine SFRO exhibits the high degree of cationic disordering $(\sim 15 \%$ from XRD result), all of the profiles have a strong resemblance, confirming that SFRO sample originally exhibits a well-ordered $\mathrm{Fe} / \mathrm{Re}$ structure even in SFRO sample holding a large amount of $\mathrm{Fe} / \mathrm{Re}$ disordering.

Although a well-ordered $\mathrm{Fe} / \mathrm{Re}$ structure is typically observed in both samples, we can notice several defective structures associated with $\mathrm{Fe} / \mathrm{Re}$ ordering, mainly in the pristine SFRO sample, which was predicted from XRD results. First, the AS defects were observed, as shown in Figure $2 \mathrm{a}$, where the Fe atomic columns indicated by the red and green arrows exhibit higher intensities than that of the one indicated by the yellow arrow. Owing to the sensitivity of the HAADF-STEM image to the atomic number, the unusually strong intensity of the Fe atomic column directly indicates the occurrence of the AS defects. To quantitatively confirm the AS defects, HAADF-STEM image simulation was performed as a function of the degree of exchange
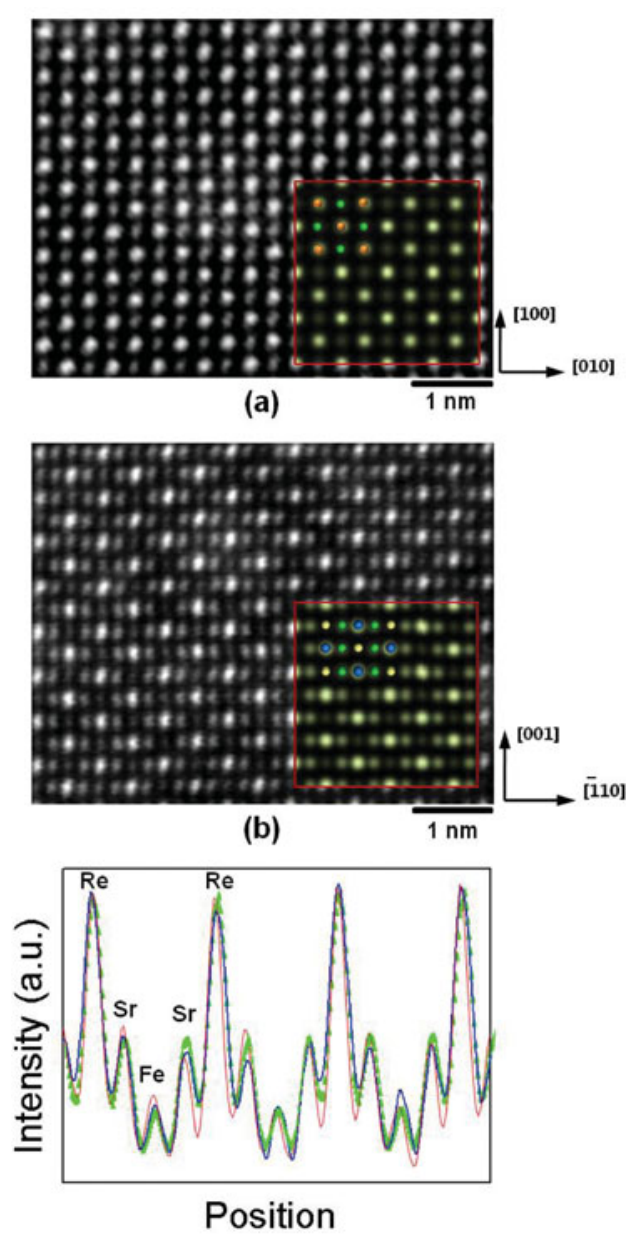

(c)

Figure 1. High-angle annular dark-field scanning transmission electron microscopy (HAADF-STEM) images from the pristine SFRO sample perpendicular to the (a) [001] and (b) [110] crystallographic axes. The insets represent the simulated HAADF-STEM images. The atomic arrays in the SFRO are superimposed on each inset ( $\mathrm{Sr}$, green; Re/Fe, orange; Fe, yellow; and Re, blue). c: Intensity profiles along direction from HAADF-STEM images of the pristine (red) and Re excess (blue) through [110] projection. Green symbols indicate the intensity profile from the simulated image.

between Fe and Re sites. Figures $2 \mathrm{~b}-2 \mathrm{~d}$ provide a series of simulated HAAD-STEM images in the [110] projection when the Fe-Re exchange occurs at 10,15 , and $20 \%$, respectively. The relative intensity of the Fe atomic column indicated by the yellow arrow in Figure $2 \mathrm{a}$ is comparable to the one from the simulated HAADF-STEM image with $10 \%$ $\mathrm{Fe}-\mathrm{Re}$ exchange (Fig. 2b). The profiling behaviors of the $\mathrm{Fe}$ columns indicated by the green and red arrows seem to exhibit more of AS defects as they are in a good agreement with those of the simulated cases at 15\% (Fig. 2c) and 20\% (Fig. 2d) Fe-Re exchanges, respectively. Second, we are able to directly confirm the APB, which supposedly hindered the movement of the magnetic domain wall in the double perovskite oxides (Yin et al., 2000; Navarro et al., 2001). $\mathrm{APB}$ is regarded as the boundary area at which $\mathrm{Fe} / \mathrm{Re}$ ordering seems to be impeded, as can be seen in Figure 3a. 


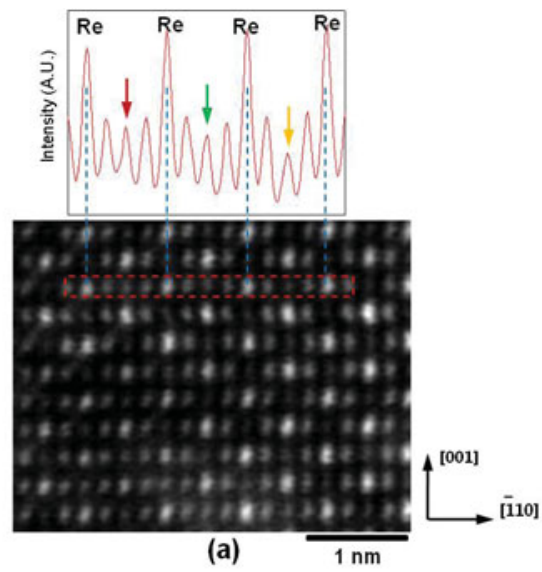

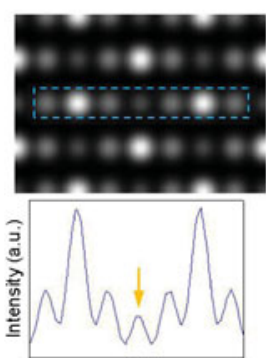

(b)

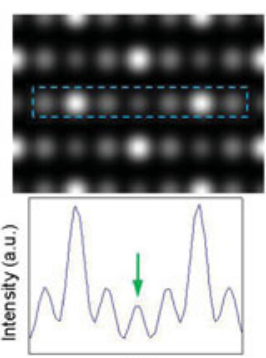

(c)

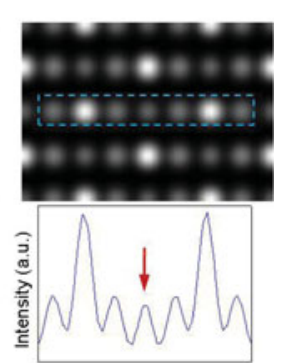

(d)
Figure 2. a: High-angle annular dark-field scanning transmission electron microscopy (HAADF-STEM) image of the pristine SFRO and the intensity profile of the atomic columns indicated by the red rectangle. The simulated HAADF-STEM images of SFRO having (b) $10 \%$, (c) $15 \%$, and (d) $20 \% \mathrm{Fe} / \mathrm{Re}$ disordering and the intensity profiles from each image.
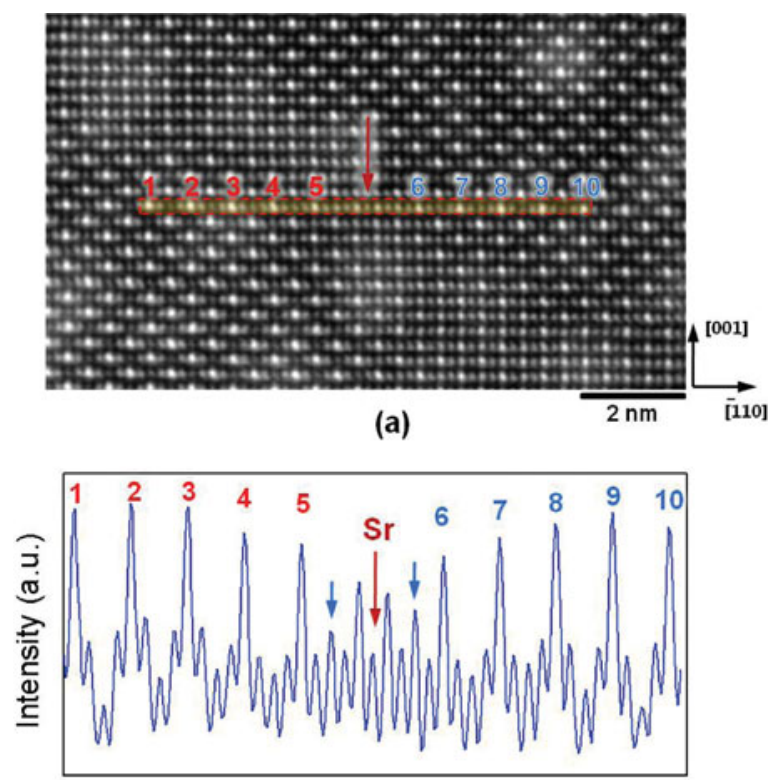

Position

(b)

Figure 3. a: High-angle annular dark-field scanning transmission electron microscopy image of Re-excess SFRO showing the atomic structure of the antiphase boundary (APB) and (b) the intensity profile along [i110] direction across the APB.

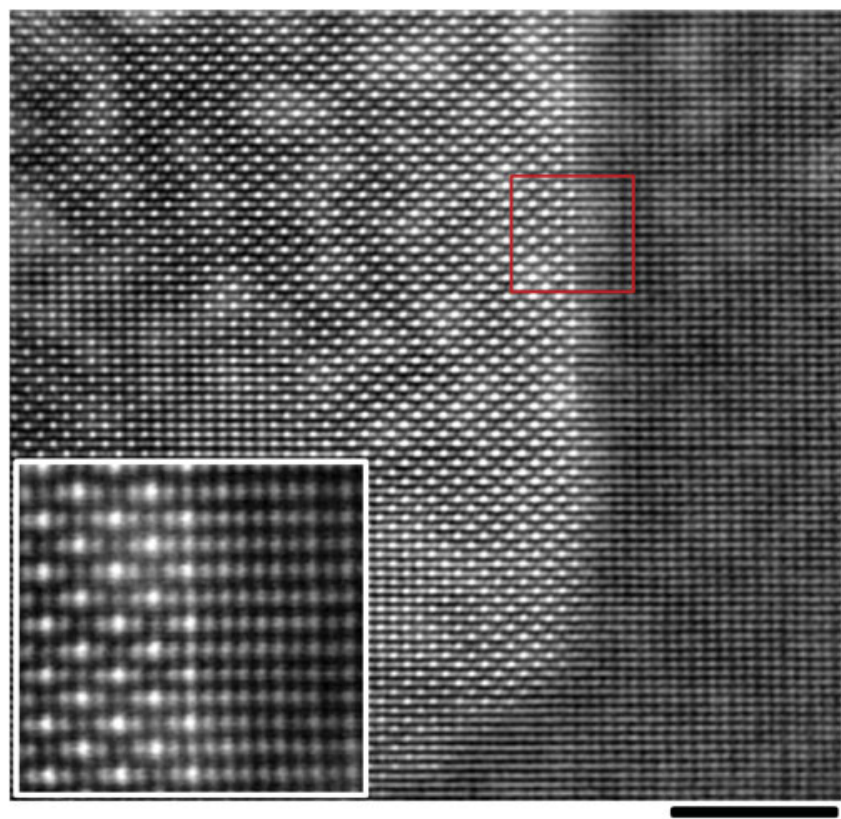

$5 \mathrm{~nm}$

Figure 4. High-angle annular dark-field scanning transmission electron microscopy image from the pristine SFRO showing the well-ordered structure of Fe/Re (left side) and Re-deficient phase (dark and right side). Inset is the enlarged image of the red square.

Figure $3 \mathrm{~b}$ shows the profiled intensity of the atomic column, indicated by the red rectangle in Figure 3a, and shows that the intensity profile is quite symmetric around the Sr atom indicated by the red arrow. In this APB region, the intensities of the Re columns (column \#4-column \#7) are found to gradually decrease along with the increased intensity of the Fe columns (blue arrows). This indicates that an APB region is correlated with the $\mathrm{Fe} / \mathrm{Re}$ cationic disordering; that strong antiferromagnetic interaction of $\mathrm{Fe}-\mathrm{O}-\mathrm{Fe}$ at APB can occur; and furthermore that the magnetic spin of Fe can be inverted across the APB. Finally, we briefly mention the Re-deficient phase, which exists only in the pristine SFRO despite the low observed frequency. The Re-deficient phase appears on the right in the dark portion of Figure 4. It is most likely $\mathrm{SrFeO}_{3}$ that has retained the antiferromagnetic Fe-O-Fe order (Mathi Jaya et al., 1991; Falcon et al., 2002). The source material for $\mathrm{Re}, \mathrm{ReO}_{3}$, is highly volatile and therefore the formation of Re-deficient phase can be inhibited by adding $15 \mathrm{wt} \%$ Re.

\section{CONCLUSIONS}

We have successfully controlled the Fe/Re ordering structure and the subsequent magnetic saturation as a function of Re source metal and elucidated a local configuration of $\mathrm{Fe} / \mathrm{Re}$ ordering in AS defects and APB via the direct imaging of individual atomic columns in aberration-corrected STEM along with the HAADF-STEM image simulations. The $\mathrm{Fe} / \mathrm{Re}$ ordering structure in SFRO can be directly identified through the [110] crystallographic axis, and a high degree of Fe/Re ordering was observed in both pristine 
and Re-excess SFRO, although AS defects were apparently observed in some of the pristine SFRO. This atomic-scale analysis suggests that the frustration of $\mathrm{Fe} / \mathrm{Re}$ ordering is accompanied by the appearance of a local distribution of AS defects and APBs rather than the homogeneous distribution of AS defects in the whole area of double perovskites.

\section{ACKNOWLEDGMENTS}

This work was supported by the National Research Foundation (NRF) of Korea through a grant provided by the Korean Ministry of Education, Science \& Technology (MEST; No. 2008-00664).

\section{REFERENCES}

Balcells, L.I., Navarro, J., Bibes, M., Roig, A., Martinez, B. \& Fontcuberta, J. (2001). Cationic ordering control of magnetization in $\mathrm{Sr}_{2} \mathrm{FeMoO}_{6}$ double perovskite. Appl Phys Lett 78(6), 781-783.

Batson, P.E., Dellby, N. \& Krivanek, O.L. (2002). Sub-ångstrom resolution using aberration corrected electron optics. Nature 418(6898), 617-620.

Choi, S.-Y., Chung, S.-Y., Yамамото, T. \& Ikuhara, Y. (2009). Direct determination of dopant site selectivity in ordered perovskite $\mathrm{CaCu}_{3} \mathrm{Ti}_{4} \mathrm{O}_{12}$ polycrystals by aberration-corrected STEM. Adv Mater 21(8), 885-889.

Chung, S.-Y., Choi, S.-Y., Yamamoto, T. \& Ikuhara, Y. (2009). Orientation-dependent arrangement of antisite defects in lithium iron(II) phosphate crystals. Angew Chem Int Ed 48(3), 543-546.

Falcon, H., Barbero, J.A., Alonso, J.A., Martinez-Lope, M.J. \& Fierro, J.L.G. (2002). $\mathrm{SrFeO}_{3-\delta}$ perovskite oxides: Chemical features and performance for methane combustion. Chem Mater 14(5), 2325-2333.

FAng, Z., Terakura, K. \& Kanamori, J. (2001). Strong ferromagnetism and weak antiferromagnetism in double perovskites: $\mathrm{Sr}_{2} \mathrm{FeMO}_{6}(\mathrm{M}=\mathrm{Mo}, \mathrm{W}$, and Re). Phys Rev B 63 (18), 180407-1-4.

García-Hernádez, M., Martínez, J.L., Martínez-Loe, M.J., CaSAIs, M.T. \& Alonso, J.A. (2001). Finding universal correlations between cationic disorder and low field magnetoresistance in FeMo double perovskite series. Phy Rev Lett 86(11), 2443-2446.

Haider, M., Uhlemann, S., Schwan, E., Kabius, B., Rose, H. \& URBAN, K. (1998). Electron microscopy image enhanced. $\mathrm{Na}$ ture 392(667B), 768-769.

Jia, C.L., Mi, S.-B., Urban, K., Vrejoiu, I., Alexe, M. \& Hesse, D. (2008). Atomic-scale study of electric dipoles near charged and uncharged domain walls in ferroelectric films. Nat Mater 7(1), 57-61.

Kobayashi, K.I., Kimura, T., Sawada, H., Terakura, K. \& Tokura, Y. (1998). Room temperature magnetoresistance in an oxide material with an ordered double-perovskite structure. Nature 395(15), 677-680.

Lindén, J., Yamamoto, T., Karppinen, M., Yamauchi, H. \& PiETARI, T. (2000). Evidence for valence fluctuation of $\mathrm{Fe}$ in $\mathrm{Sr}_{2} \mathrm{FeMoO}_{6}$-w double perovskite. Appl Phys Lett 76(20), 2925-2927.

Mathi Jaya, S., Jagadish, R., Rao, R.S. \& Asokamani, R. (1991). Electronic structure and magnetism of $\mathrm{SrFeO}_{3}$ and $\mathrm{SrCoO}_{3}$. Phys Rev B 43(16), 13274-13279.

Nakamura, S. \& Oikawa, K. (2003). Precise structure analysis consistent with Mössbauer quadrupole effect: A case of the ordered double perovskites $\mathrm{Sr}_{2} \mathrm{FeMO}_{6}(\mathrm{M}=\mathrm{Mo}$ and Re). J Phys Soc Jpn 72(12), 3123-3127.

Navarro, J., Balcells, L.I., Sandiumenge, F., Bibes, M., Roig, A., Martinez, B. \& Fontcuberta, J. (2001). Antisite defects and magnetoresistance in $\mathrm{Sr}_{2} \mathrm{FeMoO}_{6}$ double perovskite. J Phys: Condens Matter 13(37), 8481-8488.

Nellist, P.D., Chisholm, M.F., Dellby, N., Krivanek, O.L., Murfitt, M.F., Szilagyi, Z.S., Lupini, A.R., Borisevich, A., Sides, W.H., JR. \& Pennycook, S.J. (2004). Direct sub-angstrom imaging of a crystal lattice. Science 305(5691), 1741.

Yin, H.Q., Zhou, J.-S., Dass, R., Zhou, J.-P., McDevitt, J.T. \& Goodenough, J.B. (2000). Grain-boundary room-temperature low-field magnetoresistance in $\mathrm{Sr}_{2} \mathrm{FeMoO}_{6}$ films. J Appl Phys 87(9), 6761-6763.

Zajac, D., Sikora, M., Prochazka, V., Borowiec, M., Stepien, J., Kapusta, C., Riedi, P.C., Marquina, C., De Teresa, J.M. \& IbARra, M.R. (2007). Local magnetic and electronic properties of the $\mathrm{A}_{2} \mathrm{FeM}^{\prime} \mathrm{O}_{6}\left(\mathrm{~A}=\mathrm{Ba}, \mathrm{Sr}, \mathrm{Ca}, \mathrm{M}^{\prime}=\mathrm{Mo}, \mathrm{Re}\right)$ double perovskites. Acta Physica Polonica 111(6), 797-820. 